\title{
Lumen
}

Selected Proceedings from the Canadian Society for Eighteenth-Century Studies

\section{L'Antiquité retrouvée en Amérique : les images de l'Amérindien en Apollon du Belvédère}

\section{Peggy Davis}

Volume 26, 2007

Imitation et invention au siècle des Lumières

Imitation and Invention in the Eighteenth Century

URI : https://id.erudit.org/iderudit/1012066ar

DOI : https://doi.org/10.7202/1012066ar

Aller au sommaire du numéro

Éditeur(s)

Canadian Society for Eighteenth-Century Studies / Société canadienne d'étude du dix-huitième siècle

ISSN

1209-3696 (imprimé)

1927-8284 (numérique)

Découvrir la revue

Citer cet article

Davis, P. (2007). L’Antiquité retrouvée en Amérique : les images de l’Amérindien en Apollon du Belvédère. Lumen, 26, 143-158.

https://doi.org/10.7202/1012066ar

Copyright (c) Canadian Society for Eighteenth-Century Studies / Sociéte canadienne d'étude du dix-huitième siècle, 2007
Ce document est protégé par la loi sur le droit d'auteur. L'utilisation des services d'Érudit (y compris la reproduction) est assujettie à sa politique d'utilisation que vous pouvez consulter en ligne.

https://apropos.erudit.org/fr/usagers/politique-dutilisation/ 


\section{L'Antiquité retrouvée en Amérique : les images de l'Amérindien en Apollon du Belvédère}

Depuis la découverte du Nouveau Monde, les Européens ont inlassablement comparé les indigènes d'Amérique aux peuples de l'Antiquité. Au cours de nos recherches sur les images européennes de l'Amérique de la fin du XVIII ${ }^{e}$ et du début du XIX ${ }^{e}$ siècle ${ }^{1}$, nous avons eu l'occasion de rencontrer de nombreuses estampes qui représentent à l'antique l'indigène d'Amérique. Nous proposons d'explorer ici un aspect de la projection de l'Antiquité retrouvée en Amérique, en nous concentrant sur la représentation de l'Amérindien en Apollon du Belvédère (Figure 1).

La multiplication des parallèles entre les Amérindiens et les Grecs de l'Antiquité s'inscrit, d'une part, dans un discours sur l'Amérique marqué par l'émergence, au temps des Lumières, du primitivisme et $\mathrm{d}$ 'une anthropologie. Ce discours primitiviste et anthropologique joue un rôle déterminant dans l'invention d'une Amérique construite par et pour l'Européen. D'autre part, l'essor de tels parallèles répond aussi à un discours esthétique en plein renouvellement à la fin du XVIII siècle, la représentation visuelle de l'indigène d'Amérique en Apollon du Belvédère témoignant de l'autorité dont jouit la théorie de l'imitation au sein de l'esthétique néoclassique. En nous intéressant au mirage de la résurrection de l'Antiquité en Amérique, nous verrons quels ressorts de l'invention et de l'imitation sont à l'œuvre dans cette représentation.

L'indigène américain joue un rôle primordial dans la formation de l'idée que se fait l'Europe de l'Amérique : noble ou ignoble sauvage, il incarne une figure mythique et fictive, façonnée par l'imagination et modelée en fonction de l'Européen, de ses besoins et de ses attentes.

1 Voir Peggy Davis, «Perception et invention du Nouveau Monde, L'américanisme étudié à travers les estampes françaises (1750-1850)», Québec, Université Laval, Département d'histoire de l'art, thèse de doctorat, 2003, 2 vol. 
L'idéalisation à l'antique de l'Amérindien participe du premier terme de cette polarité. C'est la simplicité vertueuse de ses mœurs qui lui a maintes fois valu d'être comparé aux anciens Grecs par les Européens en quête d'une Antiquité perdue. On pense bien sûr à l'admiration devant le «bon sauvage», érigé en modèle de vertu naturelle par les Philosophes. L'Indien d'Amérique se voit en outre attribuer des qualités morales antiques - principalement celles qu'exalte le stoïcisme, mais aussi l'héroïsme guerrier et le sacrifice de soi - qui ont contribué à façonner une image de l'Amérindien animé par l'amour de l'indépendance et de la liberté. À ces éléments communs aux peuples de l'Antiquité et aux Indiens d'Amérique s'ajoute la maîtrise des arts de la guerre et de la chasse. Par ailleurs, les aristocrates européens aussi s'attribuaient volontiers toutes ces qualités et, tandis que leurs valeurs semblaient compromises par la montée de la démocratie à l'ère des Révolutions américaine et française, ils croyaient reconnaître chez certains peuples du Nouveau Monde un ethos aristocratique. Harry Liebersohn a montré comment cette image de la noblesse amérindienne était véhiculée dans les écrits des voyageurs européens et combien ces voyageurs tentaient de retrouver les vertus brutes et la noblesse sauvage de leurs cousins primitifs $^{2}$.

Certaines des représentations visuelles de l'Amérindien semblent évoquer tout naturellement cet ethos à la fois antique et aristocratique qu'on lui attribuait. Le Noble Indien de la nation Ottawa gravé par Duflos (Figure 2) est l'une des 264 planches qui ornent son Recueil d'estampes ${ }^{3}$, dans lequel le texte est quasi absent. Il est intéressant toutefois de signaler que cet inventaire des nations existantes commence par une présentation des rois d'Europe et se termine par les peuplades primitives, auxquelles appartient ce noble sauvage. Il est vêtu du drapé à l'antique, lequel a pour effet de faire valoir sa noblesse naturelle, et adopte la pose dynamique de l'Apollon du Belvédère, le pied droit en avant, la tête déviée vers l'épaule gauche et tenant l'arc d'une main. L'image du Noble Indien de la nation Ottawa participe de manière éloquente à l'invention d'un Amérindien noble et rappelant l'antique, en traduisant le désir de voir l'aristocratie primitive restaurée et l'Antiquité ressuscitée en Amérique.

2 Harry Liebersohn, Aristocratic Encounters : European Travelers and North American Indians in the New World, Cambridge, Cambridge University Press, 1998.

3 Duflos le jeune, Recueil d'estampes représentant les grades, les rangs $\mathcal{E}$ les dignités, suivant le costume de toutes les nations existantes [...], Paris, Duflos, 1780. 
L'idéalisation morale et la célébration de l'ethos antique du noble sauvage participent du primitivisme à la fin des Lumières. Cette quête d'un ailleurs, à la fois temporel et géographique, traduit au fond l'insatisfaction de l'Européen face à la civilisation moderne et son désir d'un retour à une vie simple et naturelle, comme aux origines de la création. Elle témoigne aussi d'un certain intérêt «scientifique» pour l'Amérique qui s'exprime dans un discours ethnographique et anthropologique en plein essor tout au long des $\mathrm{XVIII}^{\mathrm{e}}$ et $\mathrm{XI} \mathrm{X}^{\mathrm{e}}$ siècles. En étudiant les civilisations primitives de l'Amérique, on croyait apprendre à mieux connaître les anciens Grecs, si bien qu'on multipliait les comparaisons entre le sauvage d'Amérique et celui de l'Antiquité, dans leurs usages, mœurs et coutumes. Lafitau, à cet égard, connaît une postérité indéniable ${ }^{4}$. L'intérêt pour l'Antiquité disparue et ressuscitée au Nouveau Monde pose la question des origines de l'humanité et de la civilisation et, par extension, celle de l'extinction des peuples, puisque les Amérindiens semblent voués à disparaître depuis l'arrivée des Européens sur leur territoire.

De même, la polémique américaine occupe une place capitale dans l'élaboration des théories sur le développement historique au cours de la seconde moitié du XVIII ${ }^{\mathrm{e}}$ et du XIX ${ }^{\mathrm{e}}$ siècle. De Buffon à Darwin, en passant par Rousseau et Tocqueville, les idées sur le développement de l'humanité et de la civilisation foisonnent et s'affrontent dans cette «dispute du Nouveau Monde», pour reprendre les termes d'Antonello $\mathrm{Gerbi}^{5}$, mais ces idées portent toutes la marque de l'européocentrisme. Il est à noter par ailleurs que, selon la plupart des théories du développement de l'humanité ou de la civilisation, le stade de la chasse est considéré comme antérieur à celui de l'agriculture. Par conséquent, un peuple chasseur n'en serait que plus primitif ${ }^{6}$. Si la représentation de

4 L'ouvrage de Lafitau sur les mœurs comparées des Amérindiens et des Grecs de l'Antiquité et le récit de voyage en Amérique du baron de Lahontan, qui renforce l'association entre les sauvages d'Amérique et la république antique, s'inscrivent à la suite de Montaigne dans une tendance comparatiste de la culture humaniste. Voir Joseph-Francois Lafitau, Mœurs des sauvages américains comparées aux mœurs des premiers temps, introduction, choix de textes et notes par Edna Hindie Lemay, Paris, Maspero, 1983, 2 vol. ; Andreas Motsch, Lafitau et l'émergence du discours ethnographique, Québec et Paris, Septentrion et Presses de l'Université Paris-Sorbonne, 2001 ; Louis-Armand de Lahontan, CEuvres complètes, édition critique par Réal Ouellet, avec la collaboration d'Alain Beaulieu, Montréal, Presses de 1'Université de Montréal, 1990, 2 vol.

5 Antonello Gerbi, The Dispute of the New World. The History of a Polemic, 1750-1900, trad. Jeremy Moyle, Pittsburgh, University of Pittsburgh Press, 1973, p. 117.

6 Cornelius Jaenen, «Pelleteries et peaux-rouges : perceptions françaises de la Nou- 
l'Autre traduit le plus souvent la quête du Même, on concevra que c'est par la comparaison avec les peuples de l'Antiquité que l'Amérindien devient plus familier et donc plus susceptible de susciter l'intérêt de l'Européen.

Dans ce contexte, l'Apollon du Belvédère, en sa qualité d'auguste chasseur et canon de beauté masculine, apparaît comme le modèle par excellence de représentation de l'Amérindien. La figure idéalisée, fantasmée et inventée du sauvage, figure au demeurant étrangère dont on ne dispose d'aucun modèle en Europe, trouve son incarnation visuelle dans la plus célèbre des sculptures antiques. Autrement dit, l'invention de l'Amérindien et l'imitation de la statuaire antique se combinent pour offrir l'exemple d'une projection du Même sur l'Autre.

La représentation visuelle de l'indigène américain est investie d'éléments primitivistes liés à la grandeur antique et souscrit aux valeurs esthétiques du néoclassicisme dans lequel prédominent les théories de l'imitation et du beau idéal. Atteindre au beau idéal commande l'étude de la nature à travers l'art des Anciens, lequel résulte de la synthèse des plus belles parties de la nature. À cet égard, on connaît les prescriptions de Winckelmann, l'historien de l'art allemand du XVIII ${ }^{e}$ siècle, invitant les artistes à imiter la statuaire grecque dans le but d'obtẹnir la forme humaine idéale, plutôt que de simplement copier la nature. Bref, l'observation de la nature, par essence imparfaite, doit être validée par la référence à un modèle admis de perfection, l'art antique.

Dans le cas qui nous intéresse, la statuaire antique se présente donc comme le meilleur raccourci permettant de donner une forme, une apparence visuelle, au noble sauvage dont on idéalise les qualités morales. Au reste, l'observation directe de la nature était en soi quasi impossible, puisque l'indigène américain demeurait presque toujours inaccessible aux artistes européens ${ }^{7}$. L'Européen du XVIII ${ }^{e}$ siècle ne pouvait donc percevoir l'Amérindien autrement qu'à travers des filtres déformants qui en ont nourri et construit l'idée.

velle-France et ses peuples indigènes aux $\mathrm{XVI}^{\mathrm{e}}$, XVII ${ }^{\mathrm{e}}$ et $\mathrm{XVIII}{ }^{\mathrm{e}}$ siècles", Recherches amérindiennes au Québec, 13, 2, 1983, p. 111.

7 On notera au passage que, même lorsque des Amérindiens ont été amenés en France dans la première moitié du XIX ${ }^{\mathrm{e}}$ siècle, les images et les écrits qu'ils ont suscités demeurent fortement empreints du fantôme de l'art antique. On pense, par exemple, à Eugène Delacroix et George Sand qui croyaient reconnaître, chez ces Amérindiens à Paris, les marbres de Phidias et les guerriers de l'Antiquité grecque ressuscités. Voir notre thèse de doctorat, op. cit., vol. I, chap. 4, «L'invention de l'Indien d'Amérique : noble ou ignoble sauvage», en particulier p. 228-238. 
Les ethnographes et les voyageurs quant à eux, bien qu'ils se prétendent objectifs, présentent un Amérindien imaginaire qui traduit leurs attentes et leurs préconceptions face à l'Amérique. Leurs ouvrages sont illustrés de portraits qui s'appuieraient sur des sources documentaires authentiques, voire sur une observation simple et directe des indigènes. Les estampes démontrent pourtant que les illustrateurs, n'ayant jamais vu d'Amérindiens, n'en ont qu'une connaissance superficielle qui se limite bien souvent aux costumes et aux accessoires. Leurs images traduisent surtout une distorsion dans la représentation qu'ils en offrent et qui répond aux conventions de l'art européen.

Le Sauvage du Canada gravé par Mixelle d'après Desrais (Figure 3) se résume à un nu masculin arborant la pose et l'attitude de l'Apollon $d u$ Belvédère, et paré des armes et attributs amérindiens. Cette planche illustre le quatrième tome des Costumes civils actuels de tous les peuples connus, dessinés d'après nature, un ouvrage de Jacques Grasset de Saint-Sauveur. Ailleurs, dans ses Voyages pittoresques dans les quatre parties du mondeGrasset multipliait les entreprises éditoriales et les rééditions de ses propres ouvrages ${ }^{9}$ - il écrit : «Les Sauvages du Canada aiment la liberté, sont difficiles à civiliser, aiment les combats, et redoutent peu la mort ${ }^{10}{ }^{\prime}$. On retrouve ici formulée la perception française du Sauvage altier et indépendant, en guerrier farouche et stoïque. «L'Iroquois, qui semble être la souche de tous ces peuples ${ }^{11} »$, comme l'écrit Grasset, devient en quelque sorte le prototype de l'Amérindien, l'ancêtre commun et originel de tous les indigènes d'Amérique, le digne héritier des sauvages de l'Antiquité.

8 Jacques Grasset de Saint-Sauveur, Costumes civils actuels de tous les peuples connus, dessinés d'après nature, gravés et coloriés, accompagnés d'une Notice historique sur leurs coutumes, mours, religions, Ec. Ec., Paris, Pavard, 1788, t. IV.

9 Bernard Andrès, «Jacques Grasset de Saint-Sauveur (1757-1810), aventurier du livre et de l'estampe : première partie : la lettre de 1785 au comte de Vergennes", Les Cahiers des Dix, 56, 2002, 193-215, et «Jacques Grasset de Saint-Sauveur (17571810 ), aventurier du livre et de l'estampe : deuxième partie : du costume à la tenue d'Ève», Les Cahiers des Dix, 57, 2003, 323-352.

10 Jacques Grasset de Saint-Sauveur, Voyages pittoresques dans les quatre parties du monde, ou Troisième édition de l'Encyclopédie des voyages, contenant les principaux peuples de l'Europe, de l'Asie, de l'Afrique, de l'Amérique, et des Sauvages de la mer du Sud ; gravés et coloriés avec soin, Paris, Hocquart, 1806, t. II, p. 3.

11 Id. 
Une gravure anonyme, parue dans les Beautés de l'histoire d'Amérique (Figure 4), présente un Amérindien en tous points semblable à celui de l'ouvrage de Grasset, et cela, malgré l'annonce en page titre de « 32 nouveaux sujets de gravures». En outre, cet Amérindien appartient déjà au passé, c'est-à-dire aux temps primitifs, antérieurs à la découverte du Nouveau Monde par les Européens. L'auteur écrit : "Avant leurs relations avec les Européens, les Indiens du Canada portaient une espèce de manteau de fourrure qu'ils laissaient flotter négligemment par derrière : leurs parties naturelles étaient couvertes d'un petit tablier. Ils portaient des espèces de bottes, et leur cou était entouré $d^{\prime}$ un collier ${ }^{13}$ ». La description textuelle de la mode vestimentaire chez les anciens Indiens du Canada demeure assez proche de l'image, à l'exception du manteau de fourrure qui s'apparente plutôt ici à un drapé antique, semblable à celui de l'Apollon du Belvédère et de l'Amérindien de Grasset. En outre, les Indiens du Canada sont présentés dans cet ouvrage de Giraud comme détestant la vie sédentaire et s'adonnant essentiellement à la chasse ${ }^{14}$. Le carquois et l'arc, les attributs essentiels de l'«amérindianité», sont là pour le rappeler.

Nous remarquons également ici une volonté $d$ 'inscrire la représentation de l'indigène dans un décor comportant un paysage, même sommaire, et dans une mise en scène, ce qui diffère des autres planches ethnographiques que nous avons vues et dans lesquelles l'indigène apparaissait simplement sur un fond neutre, sans aucune référence à l'espace. L'inscription du corps dans le paysage et dans une scène presque narrative est accentuée dans une illustration du Journal d'un voyage fait dans l'Amérique septentrionale attribué à Thomas Anbury ${ }^{15}$ (Figure 5). L'Amérindien est représenté dans la pose de l'Apollon $d u$ Belvédère mais, à la différence des autres représentations examinées, il ne porte pas les attributs traditionnels que sont le carquois et l'arc. Il tient plutôt un scalp dans la main droite et un tomahawk dans la

12 Pierre François Félix Joseph Giraud (attribué à), Beautés de l'histoire d'Amérique, d'après les plus célèbres voyageurs et géographes qui ont écrit sur cette partie du monde, Paris, Librairie d'Éducation, 1816, 2 t.

13 Ibid., t. I, p. 171. Quant à leur apparence physique, l'auteur poursuit : «Les Indiens ont un tel dégoût pour le poil, qu'on en voit beaucoup qui s'arrachent les sourcils, les cils et même les cheveux, excepté une grande touffe qu'ils laissent croître derrière la tête. Ils ornent cette touffe de grains de verre, de colifichets d'argent, et, dans les grandes occasions de plumes».

14 Ibid., p. 169.

15 Thomas Anbury (attribué à), Journal d'un voyage fait dans l'Amérique septentrionale, Paris, La Villette, 1793, 2 t. 
gauche, tandis qu'à l'arrière-plan on aperçoit d'autres guerriers en train de scalper des soldats américains.

L'auteur anglais se prête à une description détaillée de la pratique du scalp chez les Indiens de la nation mohawk, lesquels, explique-t-il, "ont été chassés de leurs demeures par les Américains, et ont joint notre armée $^{16}$ ", comme si la spoliation des Amérindiens justifiait aux yeux de l'auteur l'horreur de cette pratique. Chose certaine, l'auteur, qui se montre admiratif en évoquant leur liberté et leur indépendance par contraste avec la servitude et l'oppression des civilisés ${ }^{17}$ (trait caractéristique du primitivisme), fait valoir la chasse et la guerre comme inhérentes à leur caractère fondamental. Il écrit : «Tout Indien est chasseur, et leur manière de faire la guerre ne change que d'objet, sans changer de nature ; ils poursuivent, ils surprennent et ils tuent ceux de leur espèce, au lieu de l'animal brute ${ }^{18} \gg$.

À l'examen de ces quelques exemples, on constate une volonté de précision documentaire et ethnographique dans la description textuelle et la représentation visuelle de l'Amérindien. En revanche, le recours à la statuaire antique comme source iconographique le transforme en une figure générique et universelle. Paradoxalement donc, la couleur locale et l'universalité du Beau idéal ou, en d'autres termes, l'observation ethnographique et l'idéalisation à l'antique cohabitent dans ces représentations de l'Amérindien en Apollon.

Conformément aux théories académiques en vigueur, l'Amérindien est appréhendé comme un motif de la nature étudié à travers un chef$\mathrm{d}^{\prime} œ u v r e$ de l'art antique. La doctrine néoclassique de la fin du XVIII siècle préconisait la conciliation de deux impératifs, le retour à l'antique et le retour à la nature. On conçoit donc que les artistes se soient tournés vers la statuaire antique pour y puiser des modèles de représentation de l'Amérindien; mais comment expliquer que, parmi les œuvres célèbres de la statuaire antique, l'Apollon du Belvédère constitue la source iconographique par excellence - au lieu par exemple de l'Antinoüs $d u$ Belvédère ou de l'Hercule Farnèse?

D'abord, il convient de rappeler la célébrité de l'Apollon, qui était l'une des sculptures ancrées le plus «profondément dans la conscience

16 Ibid., t. I, p. 279. Lettre XXXVII «Du camp à Battenkill, 24 août 1777».

17 Ibid., t. I, p. 52.

18 Ibid., t. I, p. 212. Lettre XXVIII «Du camp à Button-Mole-Bay, sur le lac Champlain, 24 juin 1777». 
visuelle de l'Europe cultivée ${ }^{19}{ }^{\prime}$ depuis sa découverte au début du XVI siècle. La sculpture olympienne était connue dans tous les milieux artistiques par des copies, des moulages ou des gravures avant de devenir l'un des joyaux des saisies d'Italie rapportées par Bonaparte à Paris ${ }^{20}$. Dans la mouvance de l'essor de la recherche archéologique à Rome dans la seconde moitié du XVIII siècle, les propos dithyrambiques de Winckelmann à l'égard de l'Apollon, qui ont été traduits et largement diffusés, souvent imités, voire plagiés ${ }^{21}$, ont fortement contribué au rayonnement de cette sculpture et sont certainement responsables de sa consécration comme modèle de beauté masculine.

La sculpture, qui était scrutée dans ses moindres détails par les antiquaires, a suscité un intérêt similaire à la curiosité ethnographique des voyageurs à l'égard des Amérindiens, lesquels sont par ailleurs toujours comparés à la référence universelle, l'Antiquité. À l'instar des anciens Grecs qui, selon Winckelmann, évoluaient nus dans les gymnases, révélant ainsi une multitude de poses nobles, les Amérindiens, selon la plupart des voyageurs, vivent dans un état de quasi nudité, sont dotés d'une physionomie vigoureuse et régulière, et arborent un noble maintien. Mais si Winckelmann attribuait la perfection physique des anciens Grecs à la douceur de leur climat, on ne peut, en revanche, que s'étonner de la beauté physique des Amérindiens, qui évoluent dans les rigueurs hostiles du climat nord-américain - un climat que certains, Buffon en tête, accusent de favoriser la dégénérescence.

Si l'importance de l'Apollon, érigé en modèle de beauté masculine et fleuron de la culture occidentale, n'est pas à débattre, comment expliquer son ascendant sur la perception et la représentation européennes de l'Amérindien ? Une partie de la réponse réside dans les écrits mêmes de Winckelmann. Dans ses Réflexions sur l'imitation, l'Indien d'Amérique devient une figure homérique : «Regardez l'Indien rapide à l'affût d'un cerf : voyez comme ses humeurs se fluidifient, voyez la souplesse et la rapidité de ses nerfs et de ses muscles, voyez quelle légèreté prend toute la charpente de son corps! C'est ainsi qu'Homère nous présente ses héros et il caractérise son Achille essentiellement par la rapidité de

19 Francis Haskell et Nicholas Penny, Pour l'amour de l'antique. La statuaire gréco-romaine et le goût européen 1500-1900, trad. Fr. Lissarague, Paris, Hachette Littératures, 1999 [1981], p. 161.

20 Édouard Pommier, "Winckelmann et la vision de l'Antiquité classique dans la France des Lumières et de la Révolution", Revue de l'art, 83, 1989, 9-20, en particulier p. 17-18.

21 Francis Haskell et Nicholas Penny, Pour l'amour de l'antique, op. cit., p. 134. 
ses pieds ${ }^{22}{ }$. Parmi les nombreux passages qu'il consacre à l'Apollon $d u$ Belvédère dans son Histoire de l'art chez les Anciens, Winckelmann décrit l'instant représenté en ces termes : «Ce dieu vient de poursuivre Python contre lequel il a tendu pour la première fois son arc redoutable; dans sa course rapide, il l'a atteint, et vient de lui porter le coup mortel. Pénétré de la conviction de sa puissance, et comme abîmé dans une joie concentrée, son auguste regard pénètre au loin dans l'infini, et s'étend bien au-delà de sa victoire ${ }^{23}{ }$.

Or, en intégrant le chasseur indien à son canon de beauté et en décrivant l'Apollon du Belvédère en archer, Winckelmann semble avoir, bien malgré lui, encouragé des commentateurs à établir l'analogie entre les figures homérique et apollonienne qu'il célébrait et l'Amérindien chasseur. Si bien que Winckelmann a jugé nécessaire d'ajouter à sa description du sujet représenté une digression visant à réfuter une telle interprétation, «qui prétend reconnaître dans cette statue un Apollon chasseur $^{24}$ ». Mais malgré son argumentaire, les commentateurs continuaient de faire de l'Apollon du Belvédère le prototype de l'Amérindien chasseur et guerrier, et se plaisaient à en souligner la ressemblance.

L'auteur anglais John Shebbeare, par exemple, écrivait à propos d'un Iroquois : «The Air, Attitude, and Expression of the beauteous Statue of Apollo [...] were seen animated in this American the Instant he had discharged his deadly Shaft ${ }^{25} »$. Une telle comparaison entre les Amérindiens et la statuaire grecque était devenue un lieu commun au XVIII ${ }^{\mathrm{e}}$ siècle et, dans une perspective primitiviste, contribuait à alimenter le mythe de la résurrection de l'âge d'or de l'Antiquité européenne chez les peuples d'Amérique. À ce chapitre, il convient de citer l'anecdote mythique du peintre d'origine américaine Benjamin West qui, lors de son séjour à Rome en 1760, aurait comparé l'Apollon du Belvédère à un jeune guerrier mohawk: «My God, how like it is to a young Mohawk warrior !», se serait-il exclamé devant la célèbre sculpture antique que

22 Johann J. Winckelmann, Réflexions sur l'imitation des auvres grecques en peinture et en sculpture, trad. Marianne Charrière, Nîmes, Jacqueline Chambon, 1991, p. 18.

23 Johann J. Winckelmann, Histoire de l'art chez les Anciens, traduit de l'allemand, Paris, H. J. Jansen, an II [1794], t. II, p. 428.

24 Ibid., t. II, p. 429.

25 John Shebbeare, Lydia, or Filial Piety. A Novel, New York et Londres, Garland Publishing, 1974 [1755], vol. I, p. 3-4. Voir aussi Donald A. Grinde et Bruce E. Johansen, Exemplar of Liberty: Native America and the Evolution of Democracy, Los Angeles, American Studies Center, University of California, 1991, chap. 3, «Natural Man in an Unnatural Land. Kings from America measure England, 1600-1800». 
lui présentait le cardinal Albani ; «I have seen them often [...] standing in that very attitude, and pursuing, with an intense eye, the arrow which they had just discharged from the bow ${ }^{26}{ }$. Cette anecdote amusante, qui a été rapportée pour la première fois dans la biographie «autorisée» de West en 1816, est devenue légendaire au cours des décennies suivantes et a essentiellement contribué à réhabiliter l'américanité du jeune peintre de Pennsylvanie, dont l'esprit inculte aurait pourtant reconnu dans la plus célèbre sculpture antique rien de moins qu'un guerrier amérindien ${ }^{27}$.

En somme, la vision européenne de l'Amérindien chasseur et guerrier semblait appeler tout naturellement $l^{\prime}$ Apollon du Belvédère comme modèle de représentation. Dans les ouvrages ethnographiques, l'Amérindien est en tous points conforme aux canons classiques alors en vigueur: il arbore l'expression grave d'une indépendance fière, il est vêtu d'un simple pagne, parfois d'un drapé, qui révèle la perfection de son anatomie. Il suffisait de lui ajouter quelques détails ethnographiques (la parure, les armes, les tatouages) pour accentuer la puissance de la forme classique et obtenir l'incarnation amérindienne $\mathrm{d}^{\prime}$ un noble guerrier antique.

Le fait de puiser dans l'art antique les modèles de représentation démontre un effort des illustrateurs pour ajuster leur perception des Amérindiens à des images conventionnelles et leur difficulté à s'affranchir de ces structures formelles courantes. Ainsi les images ethnographiques, au lieu de procéder d'une attitude scientifique comme

26 John Galt, The Life and Studies of Benjamin West, Esq., President of the Royal Academy in London, Prior to his Arrival in England, compiled from Materials furnished by himself, Londres, T. Cadell \& W. Davies, 1816, p. 105-106.

27 La même anecdote est également racontée dans des termes fort similaires par William Dunlap, A History of the Rise and Progress of the Arts of Design in the United States, New York, Dover Publications, 1969 [1834], p. 47-48. Notons que Galt et Dunlap prêtent au jeune Benjamin West un discours à l'égard des Indiens mohawks qui paraît directement inspiré des Réflexions sur l'imitation de Winckelmann, en particulier le passage cité plus haut (infra n. 22) à propos de la souplesse et de l'agilité du chasseur Indien, lequel s'inscrit dans une description du régime spartiate, tenu pour responsable de la beauté du corps chez les Grecs. Cet emprunt mal dissimulé aux idées de Winckelmann nous paraît confirmer la valeur de fabulation de cette anecdote. En outre, comme le territoire mohawk ne se trouve pas dans la région pennsylvanienne, West n'aurait vraisemblablement jamais vu de guerrier de cette nation, comme l'observe à juste titre Susan Rather, «Benjamin West, John Galt, and the Biography of 1816», Art Bulletin, 86, 2, juin 2004, p. 325 et p. 341, note 9. 
le propose Bernard $\mathrm{Smith}^{28}$, ne permettraient pas plus que l'art académique de donner une représentation précise, objective et empirique des peuples non européens. Puisque la représentation ethnographique de l'Amérindien est largement tributaire d'une imagerie disponible et déformée par l'idée qu'on entretient à son égard, celui-ci paraît dès lors sous une figure imaginaire et inventée.

En conclusion, il convient de s'arrêter un instant sur la question des distorsions et des préconceptions dans la représentation de l'Autre. $\mathrm{Si}$ la vision de l'Amérindien véhiculée dans les descriptions et les images ethnographiques reflète les valeurs et la culture de leurs auteurs ${ }^{29}$, l'examen des textes et des images révèle en outre la mise en place d'une tradition qui perpétue une perception européenne de l'Amérindien: de la même manière que les auteurs s'appuient sur les témoignages de leurs prédécesseurs dans lesquels ils trouvent confirmation de leurs propres perceptions de l'Autre ${ }^{30}$, les illustrateurs recourent aux modèles préexistants de représentation de l'Amérindien à l'Antique.

La perception des Amérindiens est donc le résultat d'un phénomène d'écho, en quelque sorte, puisqu'elle est alimentée par des vagues successives de rencontres avec des Européens qui perpétuent l'analogie entre ceux-ci et les Anciens. C'est bien parce que les Européens étaient à la recherche de l'Antiquité perdue et qu'ils croyaient l'avoir trouvée en Amérique qu'on peut parler de présupposés culturels qui façonnent la perception de l'Autre ${ }^{31}$. Pour appréhender le Nouveau Monde et le rendre intelligible à l'Europe, il fallait neutraliser son caractère étranger et l'assimiler au monde connu ${ }^{32}$. Or, entre comprendre et faire comprendre, les images de l'Amérindien à l'antique portent la tension entre la proximité et la distance vis-à-vis des modèles européens. La projection de l'idéal européen de l'Antiquité ressuscitée en Amérique participe de la construction identitaire de l'Europe en redéfinition au cours de la période des révolutions, à la fin des Lumières.

\section{PEGGY DAVIS}

Université du Québec à Montréal Londres, Yale University Press, 1992, p. 26-30.

29 Cornelius Jaenen, «Pelleteries et peaux-rouges», loc. cit., p. 108.

30 Harry Liebersohn, Aristocratic Encounters, op. cit., p. 7.

31 Ibid., p. 9-10, citant les travaux de James A. Boon et de Stuart B. Schwartz.

32 Cornelius Jaenen, «Pelleteries et peaux-rouges», loc. cit., p. 108. 


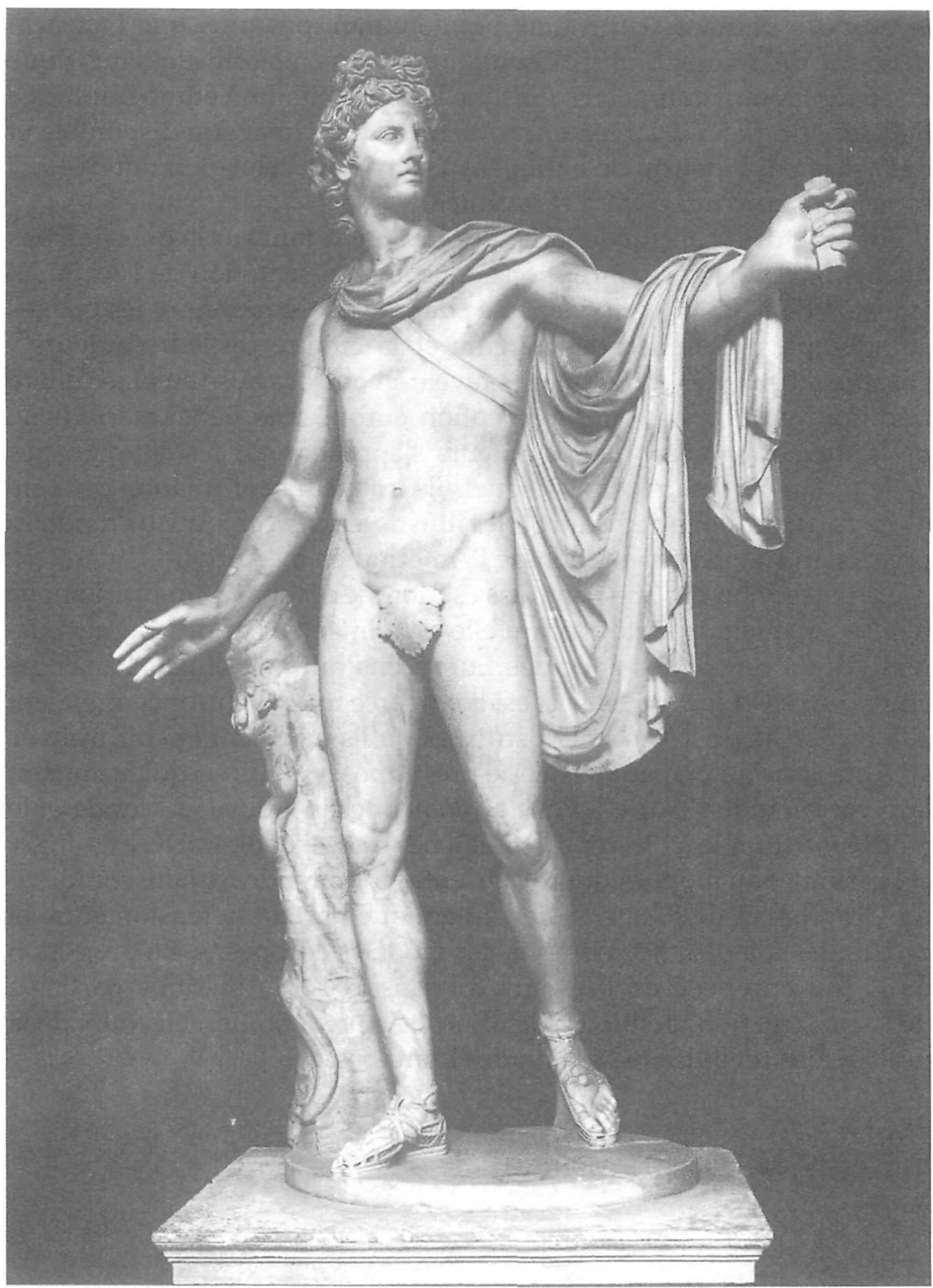

Figure 1: Apollon du Belvédère. Copie romaine d'un bronze original grec de la fin du IV siècle av. J.-C., marbre, H. 224 cm. Musée du Vatican. Les mains et l'avant-bras sculptés en 1532 ont été retirés en 1924. 


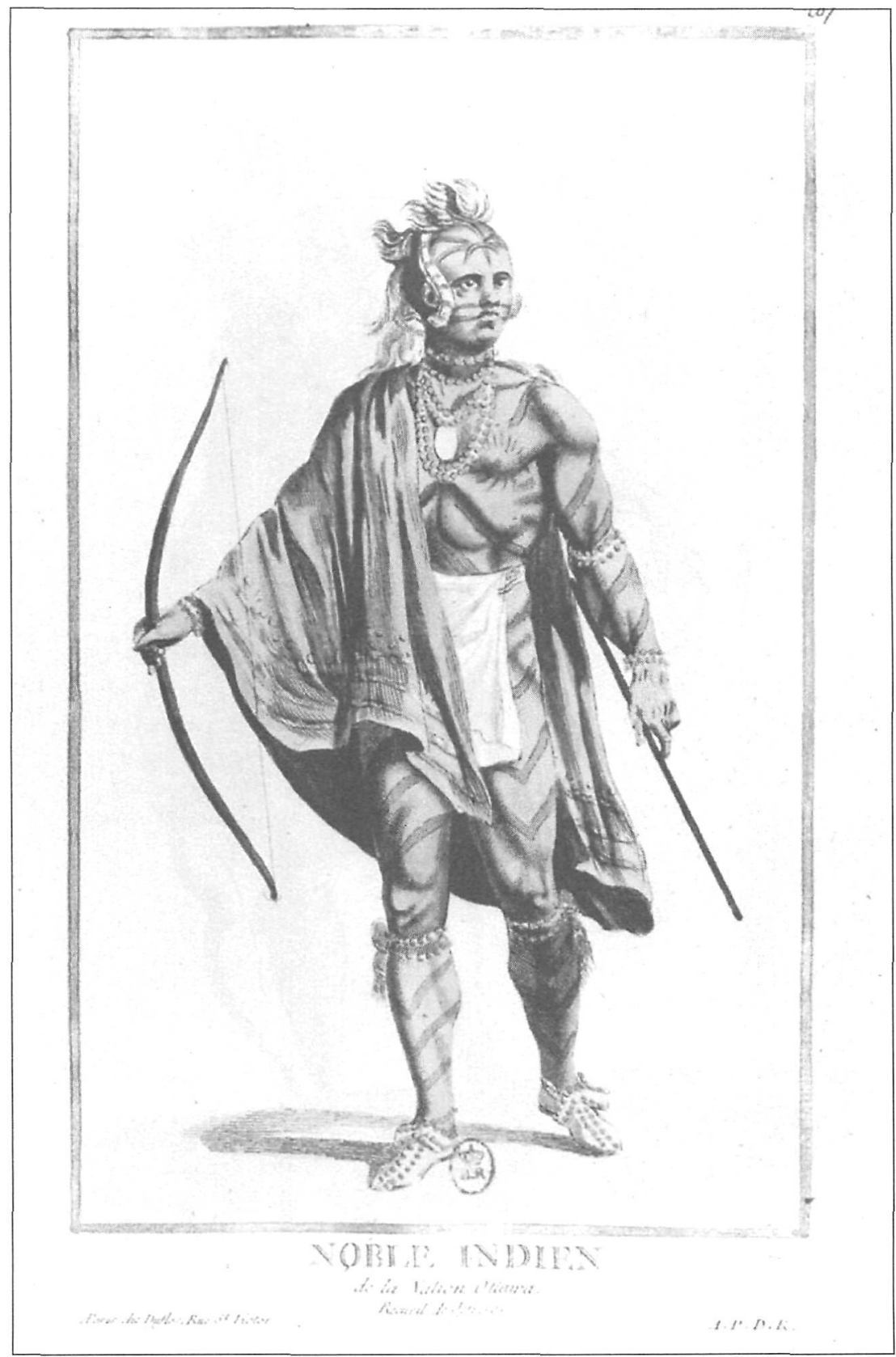

Figure 2 : NOBLE INDIEN | | de la Nation Ottawa. | | Recueil de Jefrise. Gravure coloriée de Pierre Duflos le jeune, $27,5 \times 16,5 \mathrm{~cm}$. Illustration pour P. Duflos, Recueil d'estampes représentant les Grades, les Rangs E les Dignités, suivant le costume de toutes les Nations existantes [...], 1780. BnF, cabinet des estampes. 


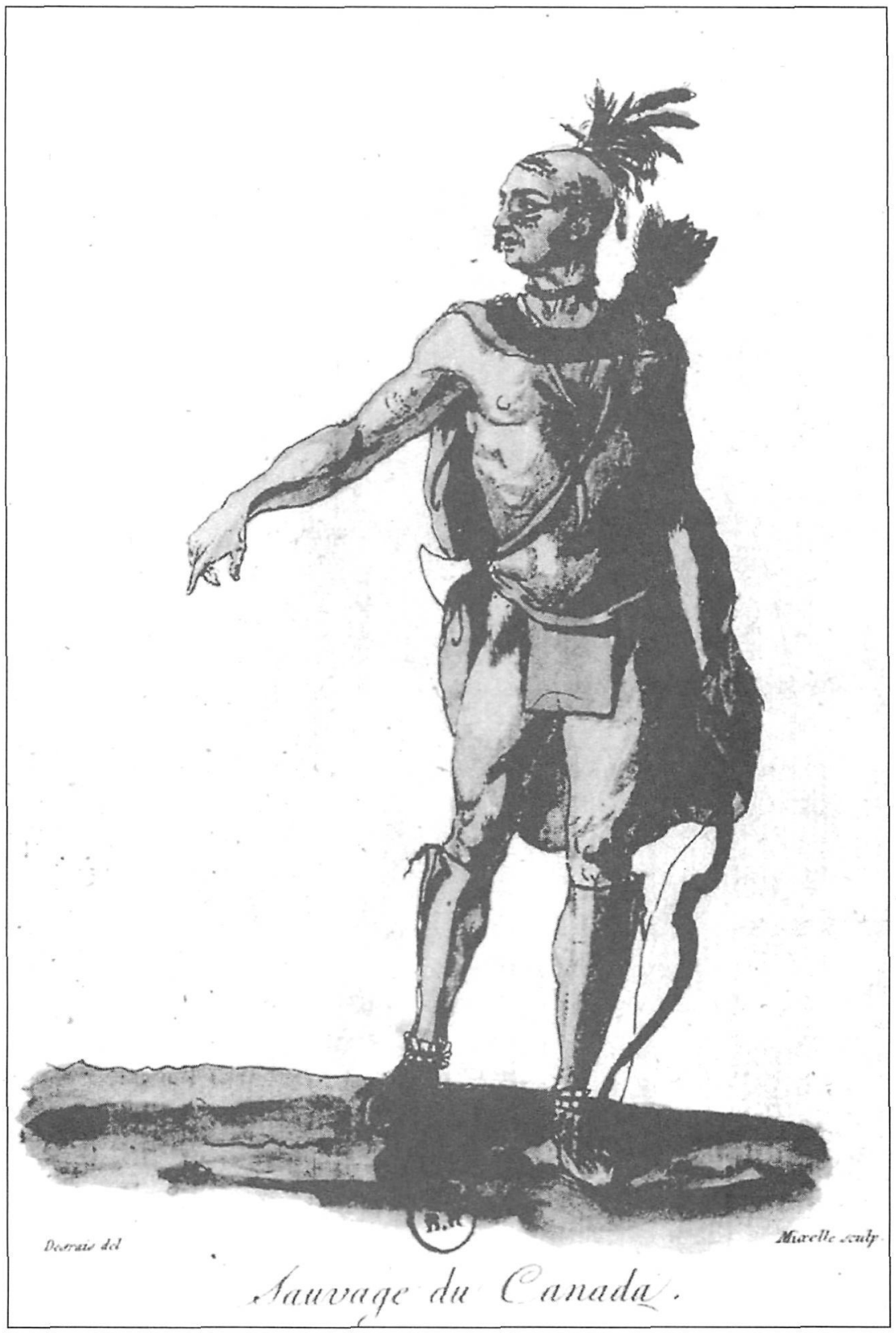

Figure 3 : Sauvage du Canada. Gravure en manière de lavis de Jean-Marie Mixelle d'après Claude Louis Desrais. Illustration pour J. Grasset de Saint-Sauveur, Costumes civils actuels de tous les peuples connus, dessinés d'après nature, gravés et coloriés [...], 1788. BnF, cabinet des estampes. 


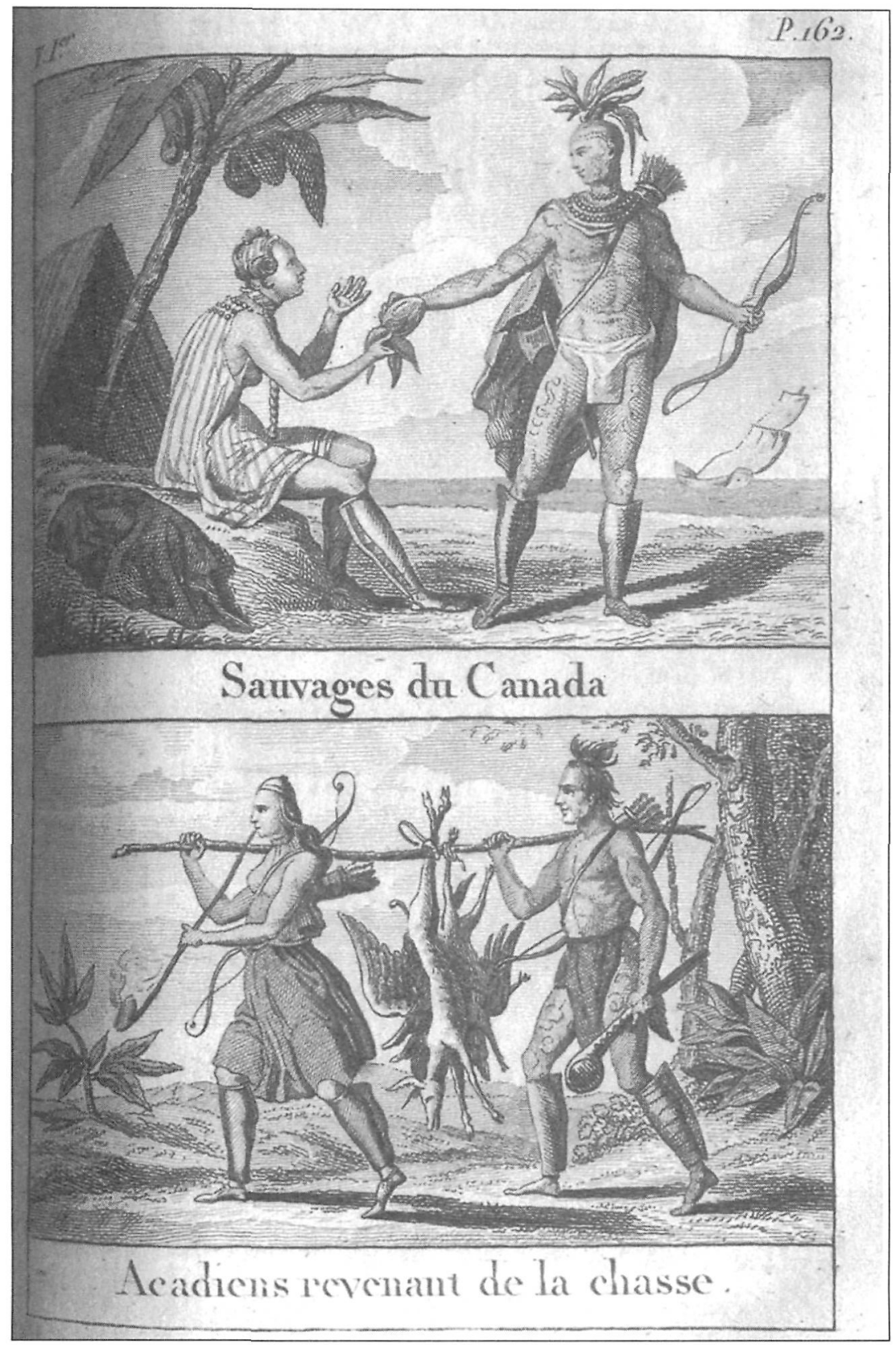

Figure 4 : Sauvages du Canada. Gravure anonyme, 6,7 x 5,1 cm. Illustration pour P. F. F. J. Giraud, Beautés de l'histoire d'Amérique: d'après les plus célèbres voyageurs et géographes qui ont écrit sur cette partie du monde, 1816. Université du Québec à Montréal, Livres rares. 


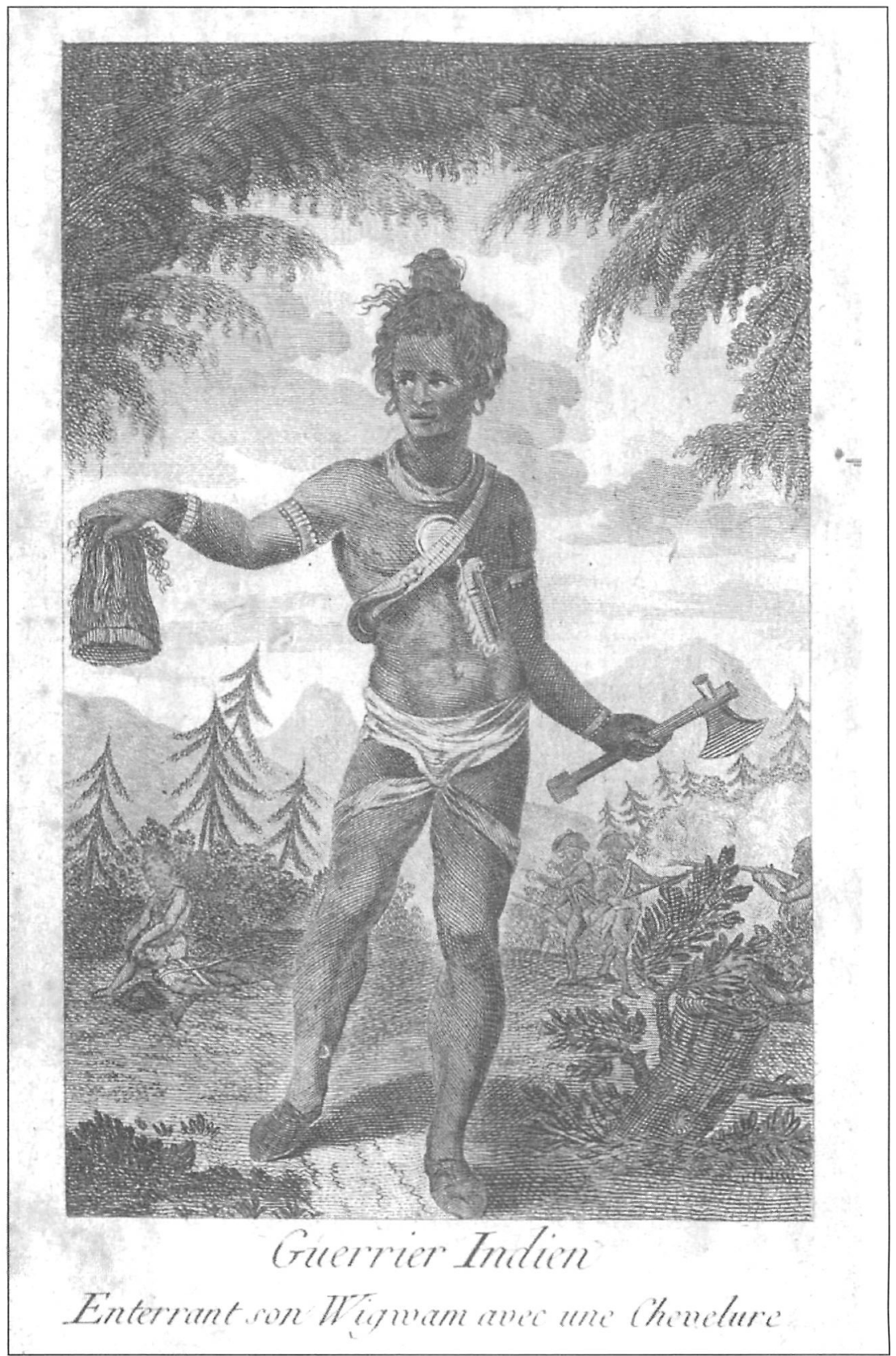

Figure 5: Guerrier Indien | | Enterrant son Wigwam avec une Chevelure. Gravure anonyme, $13,6 \times 8,9 \mathrm{~cm}$. Illustration pour [T. Anburey], Journal d'un voyage fait dans l'Amérique septentrionale, 1793. Université du Québec à Montréal, Livres rares. 